\title{
NUMERICAL RADIUS AND DISTANCE FROM UNITARY OPERATORS
}

\author{
Catalin Badea And Michel CROUZEiX
}

\begin{abstract}
Denote by $w(A)$ the numerical radius of a bounded linear operator $A$ acting on Hilbert space. Suppose that $A$ is invertible and that $w(A) \leqslant 1+\varepsilon$ and $w\left(A^{-1}\right) \leqslant 1+\varepsilon$ for some $\varepsilon \geqslant 0$. It is shown that $\inf \{\|A-U\|: U$ unitary $\} \leqslant c \varepsilon^{1 / 4}$ for some constant $c>0$. This generalizes a result due to J.G. Stampfli, which is obtained for $\varepsilon=0$. An example is given showing that the exponent $1 / 4$ is optimal. The more general case of the operator $\rho$-radius $w_{\rho}(\cdot)$ is discussed for $1 \leqslant \rho \leqslant 2$.
\end{abstract}

Mathematics subject classification (2010): 47A12.

Keywords and phrases: Numerical radius, unitary operator, unitary $\rho$-dilation.

\section{REFERENCES}

[1] T. ANDO, Structure of operators with numerical radius one, Acta Sci. Math. (Szeged) 34 (1973), 11-15.

[2] T. ANDo, C. K. LI, Operator radii and unitary operators, Oper. Matrices 4, 2 (2010), 273-281.

[3] M. Crouzeix, The annulus as a K-spectral set, to appear in Comm. Pure Appl. Anal.

[4] E. DurszT, Factorization of operators in $C_{\rho}$ class, Acta Sci. Math. (Szeged) 37 (1975), 195-200.

[5] B.-Sz. NAGY, C. FoIAŞ, Analyse harmonique des opérateurs de l'espace de Hilbert, Masson, Paris, 1967.

[6] D. D. Rogers, Approximation by unitary and essentially unitary operators, Acta Sci. Math. (Szeged) 39, 1-2 (1977), 141-151.

[7] T. Sano, A. UchiYama, Numerical radius and unitarity, Acta Sci. Math. (Szeged) 76 (2010), 581 584.

[8] J. G. Stampfl, Minimal range theorems for operators with thin spectra, Pacific Journal of Math. 23 (1967), 601-612.

[9] A. F. M. TER ElSt, Approximation by unitary operators, Acta Sci. Math. (Szeged) 54 (1990), 145-149. 